\title{
Giant Congenital Cervical Teratoma: Diagnosis, Management and Long-term Follow-up
}

\author{
Hernández Siverio N, ${ }^{1}$ López-Tomassetti Fernández, ${ }^{2}$ Arteaga González IJ, ${ }^{2}$ Troyano Luque $\mathrm{JM}^{3}$ \\ ${ }^{1}$ Department of Pediatric Surgery. \\ ${ }^{2}$ Department of Gastrointestinal Surgery. \\ ${ }^{3}$ Department of Obstetrics and Gynecology. Chief of the Gynecology Ultrasound Unit. \\ Hospital Universitario de Canarias (HUC), Canary Islands, Spain.
}

\section{SUMMARY}

Cervical congenital teratomas are rare conditions associated with abnormal prenatal development.

These tumors are usually benign; however, malignant transformation has been rarely described.

When teratomas are big, they are usually detected prenatally. In these circumstances multidisciplinary management after the delivery is necessary and the prognosis has improved provided that the airway is not obstructed and resection of the tumor is not delayed.

We present two cases of massive neonatal cervical teratoma detected prenatally by ultrasound. In one case, elective caesarean was decided at 40th week. In the other case, the child was delivered at 29th week of gestation, an airway was secured, and total resection of the tumor was performed. None of them have neurological deficit detected on long-term follow-up.

We describe our proposed management with special attention to prenatal diagnosis by ultrasound and review other series in order to provide help in decision-making.

\begin{abstract}
We report two cases of newborns with giant teratomas of the neck prenatally diagnosed. Severe polyhydramnios were present in both cases. Cesarean delivery was necessary in order to planified resuscitation and further operation of the teratoma. Prenatal diagnosis has improved the prognosis of these patients, which in different circumstances have a high morbid-mortality. At long-term follow-up both child are healthy.
\end{abstract}

Key words: Teratoma; cervicofacial teratomas; prenatal diagnosis.

\section{CASE PRESENTATIONS}

\section{Case Report 1}

A 35-year-old multiparous woman was referred at 34th week of gestation for advice about abnormal sac criteria corresponding to her gestational age.
Fetal ultrasound showed a mass tumor with mixed components measuring $90 \mathrm{~mm}$ that originated from the right neck without pulsatile components. Oral cavity was not involved. The rest of the structures of the neck were normal.

Prenatal diagnosis of benign tumor arising from the neck (teratoma or cystic hygroma) was made. Follow-up of the fetus was done every week and a cesarean section was performed at 40th week. A female healthy fetus with a huge cystic tumor on the neck was obtained. The baby weighed $3.850 \mathrm{gm}$ and Apgar score was 6/9 at 1 minute. The tumor mass originated in the right neck and had a size of $10 \mathrm{~cm}$. It was not transilluminable and had two major blood vessels surrounding it (Fig. 1). Endotracheal intubation of the newborn was not necessary and the baby was referred to a neonatal intensive care unit. Fourth day after birth surgical intervention was considered due to difficult nutrition management related to the tumor mass. At this time, the mass gross appearance had changed showing ulceration over the apex.

During surgical intervention we found that the mass was fixed at the floor of the mouth without any infiltration to the bone structure. The mass was removed and reconstruction of the mouth floor was performed.

Pathological exam revealed a $14 \times 12 \times 8.5 \mathrm{~cm}$ mass with solid components and cystic compartments full of viscous yellow fluid (Fig. 1). Histological examination of the specimen revealed an immature grade II teratoma.

Postoperative course after removal of the tumor was uncomplicated. The baby is now 16 -year-old and actually have no neurological sequels neither organ dysfunction.

\section{Case Report 2}

A 28-year-old primigravida with unremarkable past history was referred at 23rd week of gestation for advice sonographically diagnosed solid and cystic tumor in the anterior neck of the 


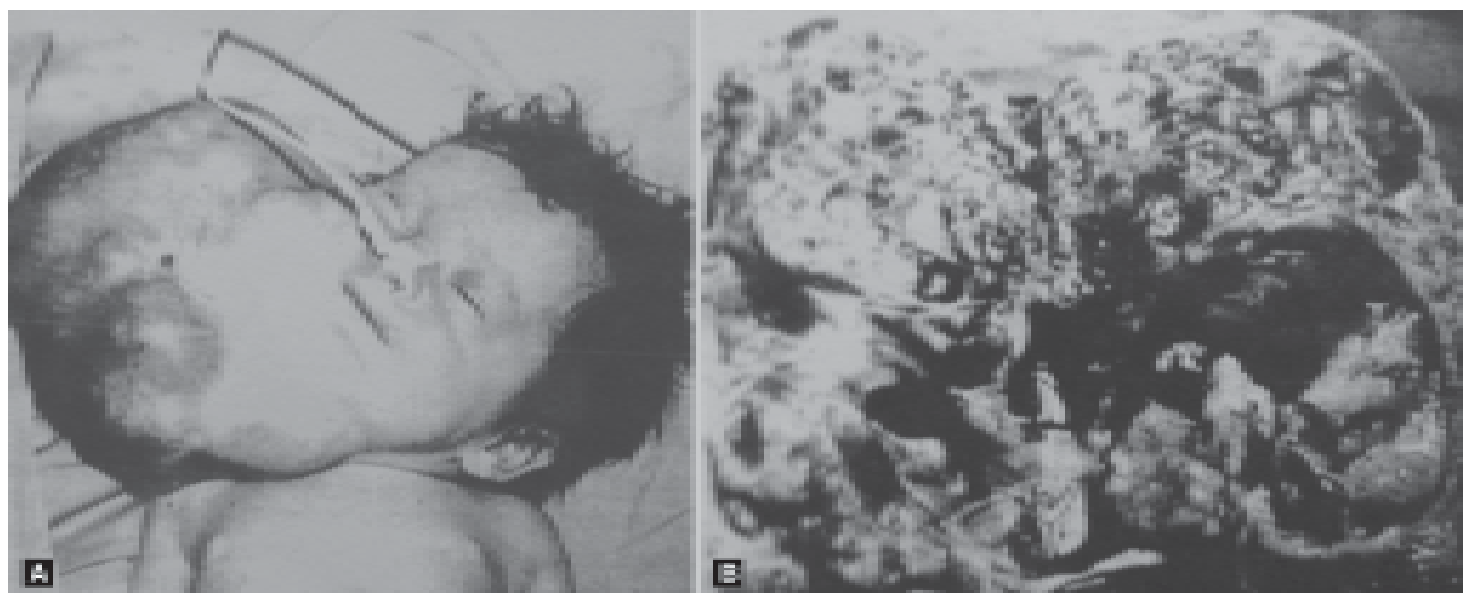

Figs 1A and B: (A) Tumor mass originating in the right neck associated with cervical hyperextension.

(B) Huge mass with solid components and cystic compartments
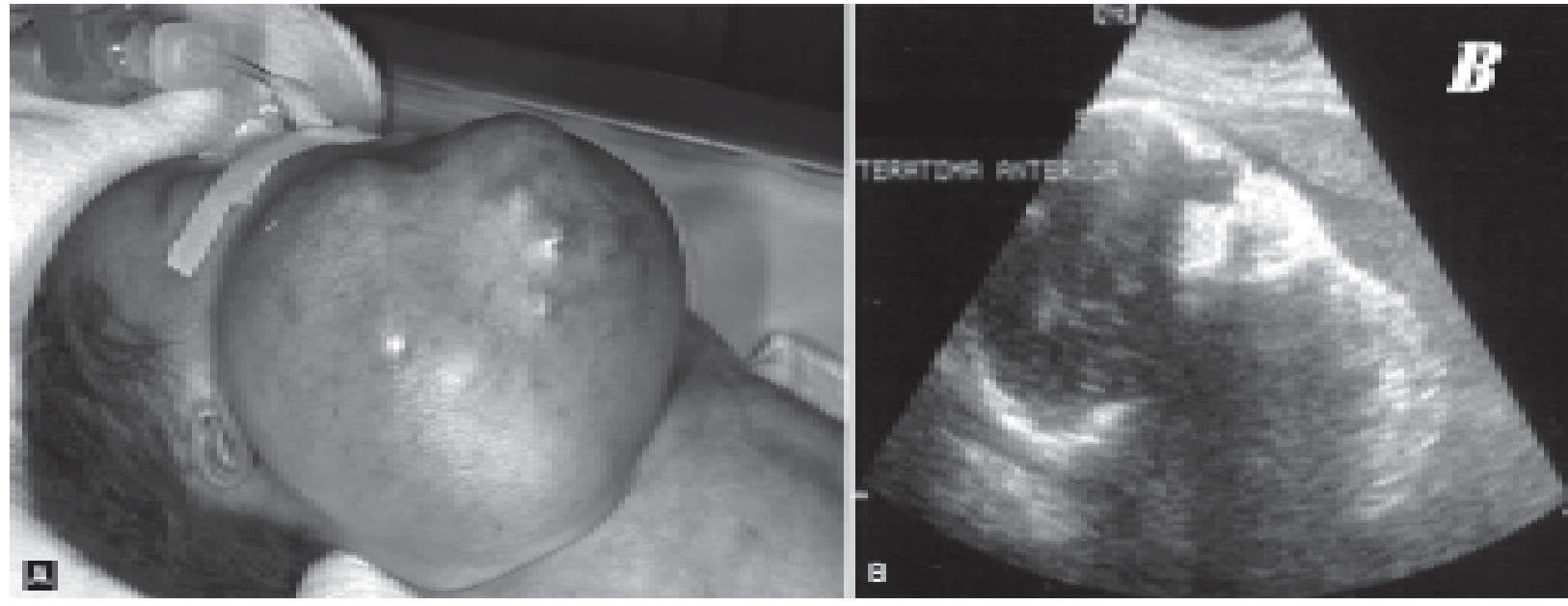

Figs 2A and B: (A) Upper airway has been adequately secured at delivery.

(B) Abdominal ultrasound shows solid tumor arising from the anterior neck

fetus associated with cervical hyperextension. Cystic areas of the tumor were predominant in the right region of the tumor (Fig. 2). The tumor mass approached the esophagus in its proximal superior region but did not involve it. Polyhydramnios was present but there was not impaired swallowing demonstrated on ultrasound. A fluid-filled stomach was present.

Transabdominal cytogenetic amniocenteses showed 46(XX) 9qh+ karyotype. Isolation of trophoblasts showed discrete rise level on basal TSH with decrease of total T3. T4 and TGBantibodies were normal.

A second ultrasound at 29th week showed polyhydramnios and rapid enlargement of the mass in the fetus neck $(9 \mathrm{~cm})$ compromising adjacent neurovascular structures. Then, we decided an elective caesarean in the 32nd week after parents were correctly informed.

A 32nd weeks estimated gestational age female was born with caesarean section. The female baby had respiratory distress with Apgar scores of 5 at 1 minute and clearly showed neck hyperextension. Nasotracheal intubation was further necessary to save baby's life (Fig. 3).

CT scan showed a non-homogenous tumor mass $(12 \times 10 \times$ 9) with solid and cystic areas. The esophagus, trachea and larynx were displaced to the patient's left with flattening of the esophageal lumen. Plain radiographs showed a soft tissue mass with calcium deposits.

Once decision to recommend operative intervention was accepted by the parents surgical intervention was carried out (second day) taking special care to avoid injuries to the thyroid gland and neurovascular structures of the neck (Fig. 4).

The pathological diagnosis was benign cystic mature teratoma. Histology showed neuroepithelium, cystic areas, calcifications, fibroelastic connective tissue and thyroid epithelial cells. The postoperative course was uneventful and at 5-year-follow-up the aesthetic result is excellent. Thyroid 


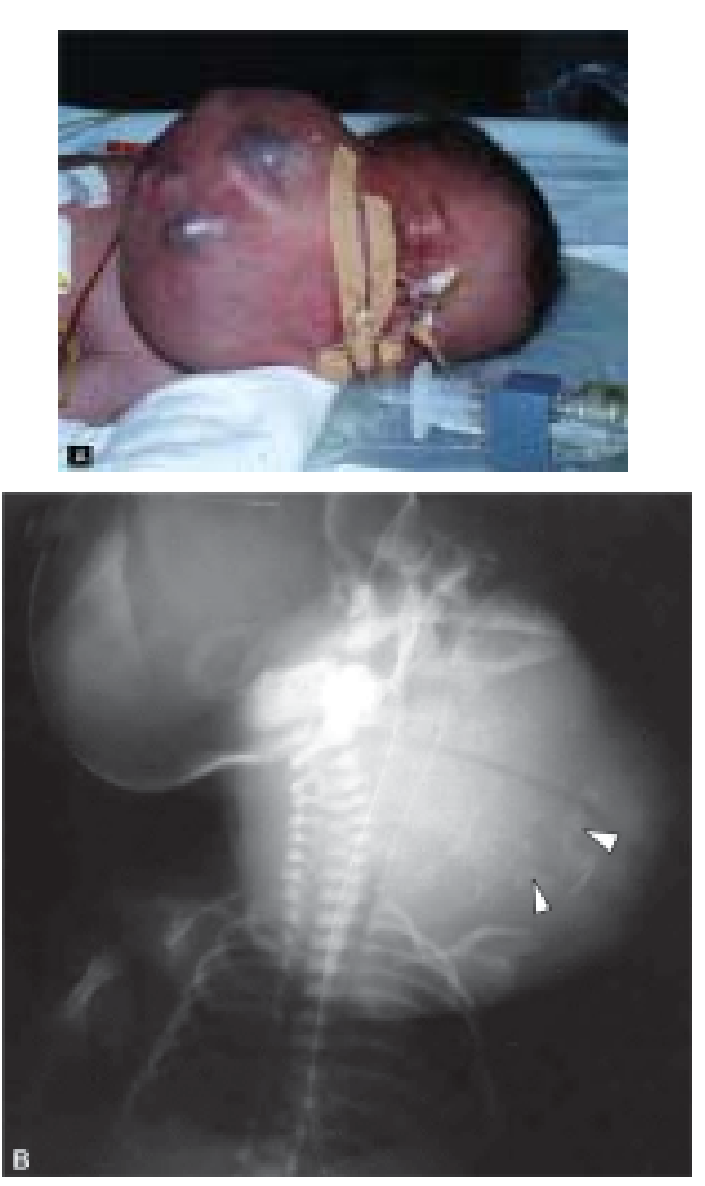

Figs $3 A$ and $B$ : (A) Cervical teratoma with cervical hyperextension. Airway has been properly secured. (B) Calcifications are shown by arrowheads in the radiographs

and parathyroid functions are normal and no neurological deficit has been developed.

\section{DISCUSSION}

Teratomas are embryonic tumors with variable degrees of differentiation composed of a variety of tissues comprising single germ cells (ectodermal, mesodermal or endodermal) and ectopic embryogenic non-germ cells. ${ }^{1}$

Despite the ongoing controversy concerning its origin, there are three major accepted theories:,2,3

1. Origin during early fetal development (loss of one twin during pregnancy).

2. Pluripotent germ cells.

3. Ectopic embryogenic non-germ cells.

Approximately 30 percent of cervical teratomas are composed of thyroid epithelium. Sometimes the thyroid gland is occupied or even substituted for teratoma tissue., ${ }^{4,5}$ This relationship to the thyroid gland has been argued to be so constant that Roediger and associates ${ }^{6}$ have postulated that all teratomas presenting in the anterior neck region arise from
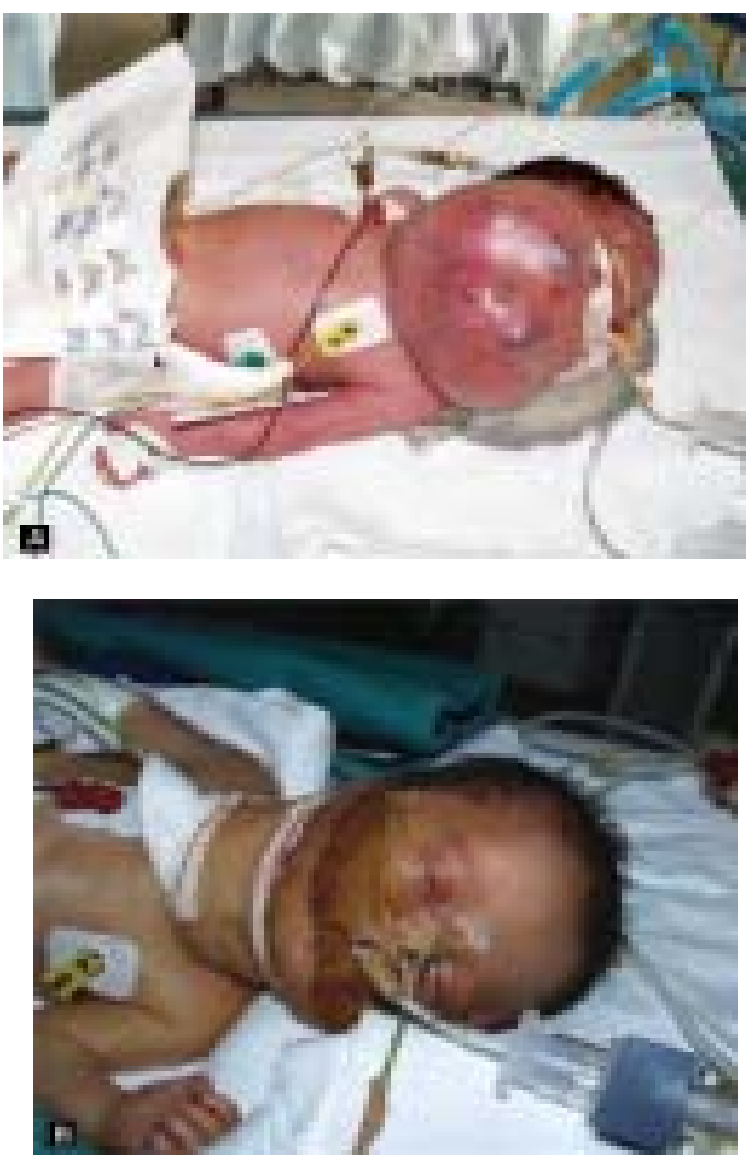

Figs 4A and B: (A) Huge tumor mass arising from the neck. (B) Postoperative photograph showing resection of the tumor mass

embryonic cells in the primitive analogue of the thyroid gland. ${ }^{7}$ Other authors do not share this opinion and regard the relationship with the gland as entirely fortuitous, in view of the extreme rarity of intrathyroid origin.

Teratomas can be broadly classified into mature or immature. Most neck teratomas are reported as mature, and malignant immature teratomas have been said to occur in approximately 5 percent of teratomas of the neck.

Mature teratomas usually contain well-differentiated tissues from the ectodermal, mesodermal, and endodermal germ cell layers, and any tissue type may be found among the tumor. Mature teratomas are benign, though some mature and immature teratomas may secrete enzymes or hormones. Immature teratomas also contain tissues from all 3 germ cell layers, but immature tissues, primarily neuroepithelial, are present. Immature teratomas can be graded from I to IV based on the amount of immature tissue found in the tumor specimen. High grade immature teratomas are associated with aggressive behavior, malignant foci and local recurrence. ${ }^{3}$ There is usually no correlation between tumor grade and patient age. 
Malignant teratomas are rare in the neonatal period; however adults are at high risk for malignancy. ${ }^{1,2,7-9}$

Common risk factors related to malignancy include: age (specially adults), high grade immature tumors, and neonatal tumors that have been not removed before two months of age. ${ }^{3}$ The prognosis of neck teratomas varies. Unless surgery is instituted without delay, the prognosis of cervical teratoma can be serious. In the past, a large proportion of patients with cervical teratomas (more than $25 \%$ of those reviewed by Silberman and Mendelson $)^{10}$ died prior to surgery.

The diagnosis of congenital cervical teratomas may be made in the antenatal, perinatal or postnatal periods. Frequently the diagnosis is made in utero by ultrasound, as is the case for our two patients. However, the diagnosis of cervical teratomas can be difficult before excision. Important improvement in the management of neonates suffering from congenital cervical teratomas and tumors in the neck has occurred during the past decade. This development is partly due to the widespread use of prenatal ultrasonography enabling prenatal diagnosis of these tumors.

Some neoplasm show specific ultrasonographic findings suggesting the differential diagnosis, but others do not. Knowledge of the presence of a neoplasm in the fetus may alter the prenatal management of a pregnancy and the mode of delivery, and facilitates immediate postnatal treatment. Our patients are a good example to show this final assessment. In both cases we decided an elective caesarean in order to avoid accidental tearing of the tumor and damage to the baby.

During pregnancy, the reason most often cited for routine ultrasound is to detect fetal anomalies. Nowadays, ultrasound is extensively used in obstetrics and gynecology. The potential benefits of ultrasound include:

1. Low cost.

2. Easily accessible.

3. Parental counseling.

4. Ultrasonography is the most innocuous and noninvasive procedure.

5. It can detect polyhydramnios (one-third of the patients; secondary to difficulty in swallowing of amniotic fluid).

6. It can detect cervical masses with solid and cystic areas (high suspicious for teratoma).

7. Ability to characterize the composition of teratomas.

8. Prenatal diagnosis permits the careful arrangement of the time, location, and mode of delivery.

9. Ability to characterize relations of the mass to the large vessels of the neck.

10. It can be useful to planned preoperatively the surgical treatment of cervical teratomas.

11. It can be useful as a guidance to localize the tracheal rings during EXIT procedure.

12. It can confirm endotracheal tube placement.

13. It may help us as assistance and guidance in surgical dissection intraoperatively.
The differential diagnoses for prenatally diagnosed neck masses are cystic hygroma, congenital goitre, thyroid masses, neuroblastoma, thyroglossal duct, branchial cleft cyst, cervical hemangioma, cervical lymphangioma, laryngocele, meningocele and macerated twin fetus. ${ }^{2,3}$ Actually, further improvements in other imaging modalities such as 3D ultrasound and ultrafast MRI has enabled us to delineate the tumor with high specificity to assure an adequate prenatal and postnatal management and parental counseling. ${ }^{11}$

There are many reports of the features of head and neck teratoma on MRI, but its use to diagnose these lesions has not been fully established. High signal intensity from fat on T1weighted images may be helpful but is not always present. Multiloculated cystic tumors with high signal on T2-weighted images may be indistinguishable from cystic hygroma.

In an elegant retrospective study aiming to evaluate the role of MR imaging to improve sonographic prenatal diagnosis of congenital anomalies the authors found that fetal MRI were most effective to study retroperitoneal pathology, meningomyelocele and visualizing the airways, brain parenchyma, and congenital brain malformation. ${ }^{11}$

Therefore, ultrafast MR imaging should be used as an adjunct to ultrasound when findings are equivocal or when the ultrasound images are difficult to interpret in the prenatal diagnosis of cervical teratomas. However, the prenatal differential diagnosis of these tumors is often difficult or even impossible (Figs 5 and 6).

Polyhydramnios can complicate 20 to 40 percent of the prenatally diagnosed cases. Over-distended uteri from polyhydramnios have been known to cause maternal complications such as ureteral obstruction. ${ }^{2,8}$ Also, the increase in uterine size due to polyhydramnios can precipitate preterm labor and delivery. Serial ultrasound examinations should be performed to monitor amniotic fluid volume. In some cases, an excess amniotic fluid level can be reduced to avoid complications. Premature labor and delivery in cases of giant cervical teratoma are common. Prenatal diagnosis allows for a management approach tailored to meet its anticipated complications. ${ }^{12}$ Airway obstruction can be life-threatening and accounts for up to 50 percent of the mortality associated with this tumors. When upper airway obstruction is anticipated, an early elective cesarean delivery should be performed (before spontaneous labor begins) and the baby should be left on fetomaternal circulation until the airway is secured. ${ }^{12}$ Tracheostomy should be reserved for airway obstruction, when orotracheal intubation is not possible. Fiberoptic bronchoscopy may obviate the need for tracheostomy in some patients if attempted at initial intubation maneuver. In our experience, one patient required nasotracheal intubation with EXIT procedure while in the other was not necessary to secure the airway. In both cases a multidisciplinary team was present at delivery. 

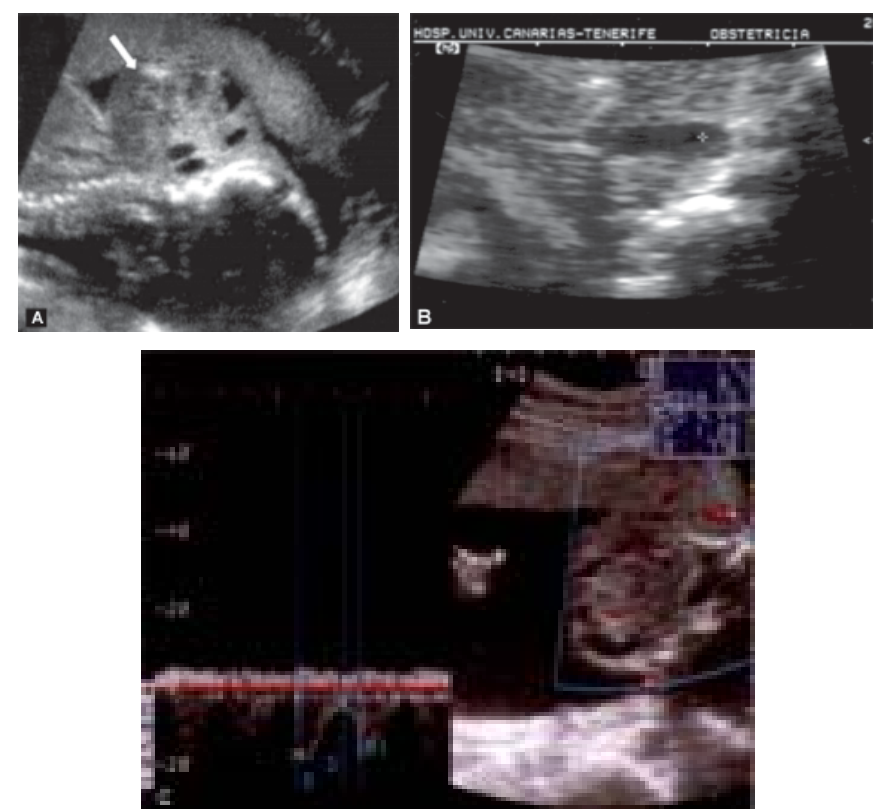

Figs 5A to C: (A) Cervical mass with solid and cystic areas (arrow); high suspicios for teratoma. (B) Cervical teratoma compressing the trachea extrinsically. Tracheal stenosis in the inferior third. Strict followup allows for a management approach to avoid airway obstruction at delivery. (C) Ability to characterise relations of the mass to the large vessels of the neck and blood supply of the tumor

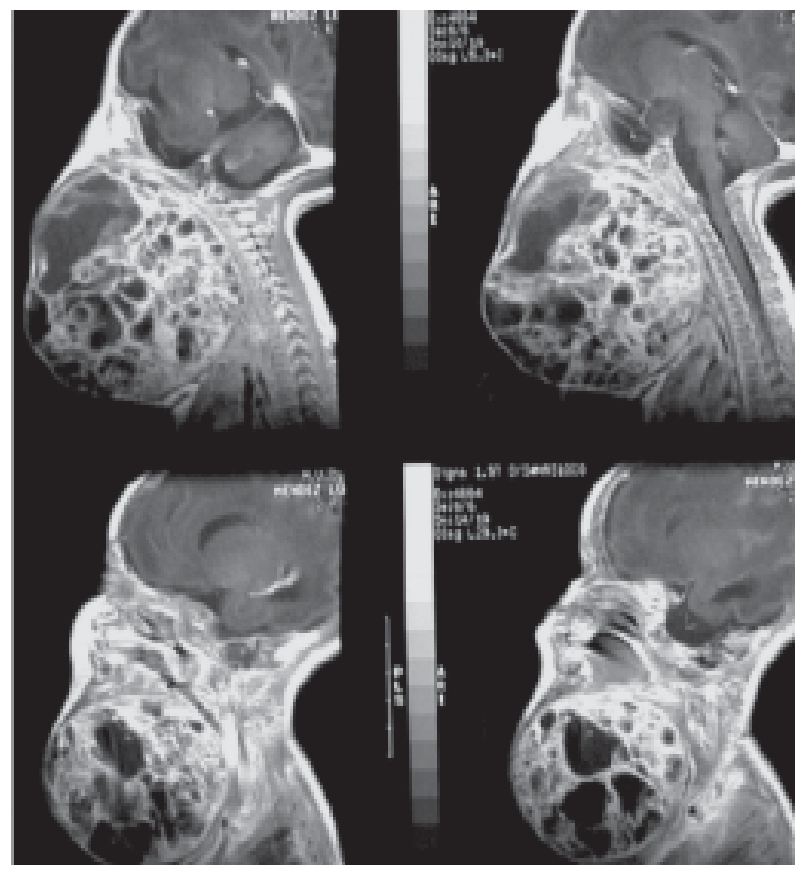

Fig. 6: Magnetic resonance of the tumor mass showed a well surrounded multiloculated cystic tumor

Two procedures has been used from the past to secure airway and avoid obstruction of the baby at birth: EXIT and OOPS procedure.
The EXIT procedure refers to Ex-utero Intrapartum. It refers to a treatment in which placental support is maintained until the airways is evaluated, surgically corrected and secure. The technique leaves an intact fetoplacental circulation and guarantees a normal fetal oxygenation while fetal airway patency is secured. To perform this procedure the head and at least one hand of the fetus are delivered and the rest of the body along with the umbilical cord and the placenta remain in utero. Only after the baby is intubated is the umbilical cord clamped and the body delivered. ${ }^{12-15}$

The OOPS procedure (operation on placental support) is a method in which umbilical cord is preserved and only clamped after the airway has been secured by intubation or by tracheotomy. Maximum uterine relaxation is important in both procedures in order to avoid separation of the placenta from the uterus and maintain fetomaternal circulation ensured. ${ }^{16}$

In addition to the OOPS and EXIT procedures, extracorporeal membrane oxygenation (ECMO), ${ }^{1}$ and cannulation of the umbilical vessels have been suggested.

Cesarean section, especially for large tumors, is indicated to avoid birth dystocia and teratoma avulsion. In rare cases in which hydrops develops earlier in gestation, open fetal surgery to remove the cervical teratoma may be necessary to save the baby. Early surgical resection of the tumor should be anticipated in cases of large cervical teratomas in order to avoid complications and malignant degeneration. Preoperative evaluation should include a chest X-ray, and CT scan of the head, neck, and chest to provide vital information regarding invasion and extension of tumor. The team approach is of utmost importance in providing an organized and coordinated care plan. An obstetrician and/or perinatologist, neonatologist, pediatric surgeon and if necessary, anesthesiologist and otolaryngologist should all be available in the delivery suite for the birth, resuscitation and possible surgical intervention of the neonate. ${ }^{17}$ At present, surgical removal offers the best approach for survival and cure. Our two babies (at just two and four days old) underwent surgical intervention to avoid further complications.

Functional and aesthetic results after surgery are usually excellent. Most common postoperative complications include recurrent laryngeal, marginal mandibular and vagus nerve damage. ${ }^{4,8,17}$ In part this is due to distortion of the neck anatomy secondary to the mass. Transient hypothyroidism and hypoparathyroidism has been described. ${ }^{8}$ Mental retardation ranging from mild to profound is probably secondary to hypoxia associated with prolonged resuscitation maneuvers.

Alpha-fetoprotein is elevated in cases of fetal neural tube defects, congenital defects, and anomalies such as cervical teratoma. Usually, a preoperative alpha-fetoprotein level is recommended. Obtaining serial beta-hCG and alpha-fetoprotein levels will allow for follow-up of the completeness of tumor excision or recurrence. 
In a recent review it was stated that the most important variable affecting long-term survival in literature cases has been successful and complete resection of the tumor. ${ }^{18}$ At long-term follow-up (at least 5 years), our patients had no evidence of local or distant recurrence on examination and radiological images.

Even though cervical teratomas are considered benign tumors in infancy, occasional malignant transformation and consequent death have been reported. Until 1998, true malignant teratomas have been reported in only 9 of 220 cases. The diagnosis of malignancy was made secondary to metastasis and/or histological evidence of germinoma, embryonal carcinoma, or choriocarcinoma. ${ }^{18}$

\section{REFERENCES}

1. Kelly MF, Berenholz L, Rizzo KA, Greco R, Wolfson P, Zwillenberg DA. Approach for oxygenation of the newborn with airway obstruction due to a cervical mass. Ann Otol Rhinol Laryngol 1990;99:179-82.

2. Kountakis SE, Minotti AM, Maillard A, Stiernberg CM. Teratomas of the head and neck. Am J Otolaryngol 1994;15:2926.

3. Carr MM, Thorner P, Phillips JH. Congenital teratomas of the head and neck. J Otolaryngol 1997;26:246-52.

4. Chowdhary SK, Chitnis M, Perold J, Lazarus C. Hypothyroidism in a neonate following excision of a cervical teratoma. Pediatr Surg Int 1998;14:212-3.

5. Hassab MH, Shadi SM, Al-Olayet YF, Al-Bassam AR. Ten years' experience in the management of cervical teratomas. Pediatr Surg Int 1996;11:536-8.

6. Roediger WE, Spitz L, Schmaman A. Histogenesis of benign cervical teratomas. Teratology 1974;10:111-8.

7. Als C, Laeng H, Cerny T, Kinser JA, Rosler H, Hausler R. Primary cervical malignant teratoma with a rib metastasis in an adult: Five-year survival after surgery and chemotherapy. A case report with a review of the literature. Ann Oncol 1998;9:1015-22.
8. Azizkhan RG, Haase GM, Applebaum H, Dillon PW, Coran AG, King PA, King DR, Hodge DS. Diagnosis, management, and outcome of cervicofacial teratomas in neonates: A Childrens Cancer Group study. J Pediatr Surg 1995;30:312-6.

9. Baumann FR, Nerlich A. Metastasizing cervical teratoma of the fetus. Pediatr Pathol 1993;13:21-7.

10. Silberman R, Mendelson IR. Teratoma of the neck: Report of two cases and review of the literature. Arch Dis Child 1960;35:159-70.

11. Breysem L, Bosmans H, Dymarkowski S, Schoubroeck DV, Witters I, Deprest J, Demaerel P, Vanbeckevoort D, Vanhole C, Casaer P, Smet M. The value of fast MR imaging as an adjunct to ultrasound in prenatal diagnosis. Eur Radiol 2003;13:153848 .

12. Hirose S, Sydorak RM, Tsao K, Cauldwell CB, Newman KD, Mychaliska GB, Albanese CT, Lee H, Farmer DL. Spectrum of intrapartum management strategies for giant fetal cervical teratoma. J Pediatr Surg 2003;38:446-50.

13. Langer JC, Tabb T, Thompson P, Paes BA, Caco CC. Management of prenatally diagnosed tracheal obstruction: Access to the airway in utero prior to delivery. Fetal Diagn Ther 1992;7:12-6.

14. O’Callaghan SP, Walker P, Wake C, Roy G, Farrell P. Perinatal care of a woman with the prenatal diagnosis of a massive fetal neck tumour (cervical teratoma). Br J Obstet Gynaecol 1997;104:261-3.

15. Hirose S, Harrison MR. The ex utero intrapartum treatment (EXIT) procedure. Semin Neonatol 2003;8:207-14.

16. Skarsgard ED, Chitkara U, Krane EJ, Riley ET, Halamek LP, Dedo HH. The OOPS procedure (operation on placental support): In utero airway management of the fetus with prenatally diagnosed tracheal obstruction. J Pediatr Surg 1996;31:826-8.

17. Sichel JY, Eliashar R, Yatsiv I, Moshe Gomori J, Nadjari M, Springer C, Ezra Y. A multidisciplinary team approach for management of a giant congenital cervical teratoma. Int J Pediatr Otorhinolaryngol 2002;65:241-7.

18. Kerner B, Flaum E, Mathews H, Carlson DE, Pepkowitz SH, Hixon H, Graham JM Jr. Cervical teratoma: Prenatal diagnosis and long-term follow-up. Prenat Diagn 1998;18:51-9. 